\title{
Réflexion de la houle sur les ouvrages :
}

\author{
modèle de houle à dissipation
}

\author{
M. HOUARI(*), P. SERGENT(**), D. DUHAMEL $(*)$ \\ Ecole Nationale des Ponts et Chaussées (*) \\ 6 et 8 avenue Blaise Pascal
}

Citée Descartes, Champs-sur-Marne 77455 Marne-la-Vallée cedex 2

Tel :0164153728Email :duhamel@lami.enpc.fr

Centre d'Etudes Techniques Maritimes Et Fluviales (**)

2, boulevard Gambetta BP 6003960321 Compiègne cedex

Tel :0344926030 Email : philippe.sergent@equipement.gouv.fr

Résumé : La réflexion de la houle sur les ouvrages est un problème particulièrement important pour l'ingénierie portuaire. Les conditions de réflexion classiques sur les ouvrages demandent la connaissance d'un angle d'incidence a priori inconnu. Afin de s'en affranchir, nous développons un modèle horizontal de houle à dissipation. La dissipation est estimée à partir de la propagation de la houle dans un bicouche composé d'une couche fluide couvrant une couche poreuse. Le modèle est testé sur une bosse triangulaire poreuse et sur une digue à talus.

\begin{abstract}
Reflection of water waves on maritime structures is an important problem of coastal engineering. Classic reflection conditions on structures require the knowledge of an angle of incidence that is a priori unknown. In order to get rid of it, we develop a horizontal dissipative model for water waves. Dissipation is assessed from the propagation of wave in a bilayer composed of a layer of fluid covering a porous bed. The model is tested on a triangular porous bar and a rubble mound breakwater.
\end{abstract}

\section{$\underline{\text { 1. Introduction }}$}

La réflexion de la houle, à l'approche des ouvrages, intéresse particulièrement l'ingénierie portuaire. Elle a un effet direct sur la stabilité des ouvrages et sur l'agitation car elle constitue l'un des principaux facteurs de dissipation de la houle dans un bassin portuaire. Le traitement de la réflexion partielle dans les codes d'agitation, basés en général sur l'équation de pente douce, reste un problème délicat qui nécessite la connaissance :

- Du coefficient de réflexion, qui représente une caractéristique globale de l'effet des ouvrages étudiés, et en particulier l'influence de paramètres tels que 
la période de houle, la cambrure de houle, la direction d'incidence sur ce coefficient,

a De l'angle d'incidence de la houle pour pouvoir appliquer correctement la condition aux limites classique de réflexion partielle.

Cet angle d'incidence de la houle étant a priori inconnu, son utilisation pose des problèmes aussi bien théoriques que pratiques. Il donc intéressant de pouvoir proposer une formulation qui n'utilise pas cet angle. C'est le cas du modèle horizontal que nous présentons ici et qui permet de traiter la réflexion partielle de la houle sur les ouvrages réfléchissants en prenant en compte la dissipation de la houle sur les enrochements.

Les ouvrages de protection des ports contre la houle sont les digues à talus, les jetées verticales ou les digues mixtes. Ils arrêtent la houle par destruction locale de son énergie ou par réflexion vers le large. Les digues à talus sont des collines de matériaux pierreux, enrochements, qui montent jusque vers la surface libre. Les digues mixtes sont constituées par une digue verticale posée sur une digue à talus constamment immergée. Nous présentons, dans ce qui suit, un modèle pour traiter l'écoulement dans ces ouvrages.

\section{$\underline{2 \text { Milieu poreux }}$}

\subsection{Equation de Forchheimer}

On considère un milieu poreux saturé composé de deux phases : le squelette rigide et le fluide saturant l'espace interstitiel. Le milieu poreux est considéré inerte, il n'y a pas de création de masse pour les deux phases. On considère aussi que le squelette est immobile et que le fluide est incompressible. Les équations de conservation de la masse et de la quantité de mouvement sont :

$$
\operatorname{div}\left(\varepsilon \vec{V}_{s}\right)=0
$$

avec $\varepsilon$ la porosité du milieu, $\vec{V}_{s}$ la vitesse interstitielle ou vitesse moyenne réelle du fluide. La quantité $\varepsilon \vec{V}_{s}$ désigne la vitesse de filtration ou vitesse d'écoulement.

$$
\frac{\partial \vec{V}_{s}}{\partial t}=-\frac{1}{\rho} \vec{\nabla}(p+\rho g z)-\vec{F}_{r}-\vec{F}_{i}
$$

où $\rho$ représente la masse volumique du fluide, $p$ la pression, $z$ la cote verticale et $\vec{\nabla}$ désigne l'opérateur gradient.

Le terme de résistance $\vec{F}_{r}$ s'exprime en fonction de la vitesse interstitielle (Ward, 1964) : 


$$
\vec{F}_{r}=\frac{v \mathcal{E}}{K} \vec{V}_{s}+\frac{C_{f} \varepsilon^{2}}{\sqrt{K}}\left|\vec{V}_{s}\right| \vec{V}_{s}
$$

où $v$ représente la viscosité, $C_{f}$ le coefficient de frottement turbulent et $K$ la perméabilité du milieu poreux.

Le terme de résistance inertielle $\vec{F}_{i}$ dépend du coefficient de masse virtuelle $C_{m}$ et de l'accélération dans le sens de l'écoulement (Sollit, 1972).

$$
\vec{F}_{i}=\frac{1-\varepsilon}{\varepsilon} C_{m} \frac{\partial \vec{V}_{s}}{\partial t}
$$

Le coefficient $C_{m}$ peut être évalué pour un grain individuel de forme régulière mais reste généralement inconnu pour un ensemble de grains formant un corps poreux. En remplaçant les expressions de $\vec{F}_{r}$ et $\vec{F}_{i}$ dans l'équation de conservation de la quantité de mouvement, on obtient l'équation de Forchheimer qui est l'équation généralement utilisée par les ingénieurs pour décrire les écoulements dans les milieux poreux sous l'action de la houle.

$$
S \frac{\partial \vec{V}_{s}}{\partial t}=-\frac{1}{\rho} \vec{\nabla}(p+\rho g z)-\frac{v \varepsilon}{K} \vec{V}_{s}-\frac{C_{f} \varepsilon^{2}}{\sqrt{K}}\left|\vec{V}_{s}\right| \vec{V}_{s}
$$

avec $S=1+\frac{1-\varepsilon}{\varepsilon} C_{m}$.

Le terme linéaire, terme de Darcy, gouverne les équations à faible nombre de Reynolds en milieu poreux assez fins. Le terme non-linéaire, terme de Forchheimer, est une extension du terme de Darcy pour des milieux poreux plus grossiers, à grands nombre de Reynolds où les phénomènes d'inertie l'emportent sur les effets de la viscosité.

\subsection{Linéarisation}

En considérant un mouvement harmonique, les termes dissipatifs, linéaire et non linéaire, de l'équation de Forchheimer sont remplacés par un terme linéaire équivalent :

$$
\frac{v \varepsilon}{K} \vec{V}_{s}+\frac{C_{f} \varepsilon^{2}}{\sqrt{K}}\left|\vec{V}_{s}\right| \vec{V}_{s} \rightarrow f \omega \vec{V}_{s}
$$

Dans cette expression, $f$ peut être assimilé à un coefficient de frottement ou d'amortissement, $\omega$ représente la pulsation du mouvement harmonique de période $T$. L'évaluation du coefficient $f$ repose sur l'hypothèse de Lorentz du travail 
équivalent qui consiste à égaliser sur une période, le travail du terme non-linéaire avec celui d'un terme linéaire.

Le terme $f$ vaut (Dingemans, 1996):

$$
f \omega=\frac{v \varepsilon}{K}\left(1+\frac{C_{f} \varepsilon \sqrt{K}}{v} \bar{f}\right)
$$

avec

$$
\bar{f}=\frac{\int_{0}^{T}\left|\vec{V}_{s}\right|^{3} d t}{\int_{0}^{T}\left|\vec{V}_{s}\right|^{2} d t}=\frac{8}{3 \pi} \sqrt{a+b}\left\{E(m)-\frac{1}{4}\left(1-\frac{b}{a}\right) K(m)\right\}
$$

et $\quad a=\frac{1}{T} \int_{0}^{T}\left|\vec{V}_{s}\right|^{2} d t, \quad b=\operatorname{Max}\left(\left|\vec{V}_{s}\right|^{2}-a\right), \quad m=\frac{2 b}{a+b} . K(m)$ et $E(m)$ représentent respectivement les intégrales elliptiques de premier et de second ordre.

\subsection{Equation pour un mouvement harmonique}

Ecrivons les solutions harmoniques :

$$
\begin{aligned}
& \vec{V}_{s}=\operatorname{Re}\left(\overrightarrow{\mathbf{V}} e^{-j \omega t}\right) \\
& p^{*}=\operatorname{Re}\left(\mathbf{P} e^{-j \omega t}\right)
\end{aligned}
$$

avec $p^{*}=p+\rho g z$

L'écriture linéaire de l'équation de conservation de la quantité de mouvement en fonction des grandeurs $(\overrightarrow{\mathbf{v}}, \mathbf{P})$ et du terme de linéarisation $f$ est :

$$
-j \omega S \overrightarrow{\mathbf{V}}=-\frac{1}{\rho} \vec{\nabla} \mathbf{P}-f \omega \overrightarrow{\mathbf{V}}
$$

que l'on peut aussi mettre sous la forme donnant la vitesse de filtration :

$$
\varepsilon \overrightarrow{\mathbf{V}}=\frac{1}{\rho j \omega} \beta \vec{\nabla} \mathbf{P}
$$


avec $\beta=\frac{\varepsilon}{S+j f}$. L'équation de conservation de la masse permet d'obtenir la relation de propagation finale :

$$
\operatorname{div}(\beta \vec{\nabla} \mathbf{P})=0
$$

Cette relation, avec les conditions aux limites sur la surface libre, le fond rigide et à l'interface du milieu poreux et du milieu fluide, définit un modèle à pression régissant la propagation d'une houle linéaire périodique en présence de milieu poreux. La résolution de cette équation, par un schéma itératif, permet de définir le champ de vitesse ainsi que la distribution de la pression dans le milieu poreux. Cette équation est commune aux deux milieux, le domaine est considéré alors comme hétérogène avec une fonction $\beta$ variable dans l'espace. La fonction est constante et égale à 1 dans le fluide, elle est variable dans le milieux poreux.

\section{Bicouche}

Soit le cas du bicouche représenté par la figure 1 et composé d'un fluide submergeant un fond poreux qui repose sur un fond rigide. Soit $O x y z$ un repère cartésien dont les axes $\mathrm{O} x, \mathrm{O} y$ définissent le plan horizontal coïncidant avec la surface libre du fluide au repos, l'axe $\mathrm{O} z$ est dirigé vers le haut. Les vecteurs unitaires normaux à l'interface fluide-poreux et au fond rigide sont notés respectivement $\vec{n}$ et $\vec{n}_{f}$, $h$ représente la profondeur de la couche fluide et $h_{f}$ la profondeur totale du domaine.

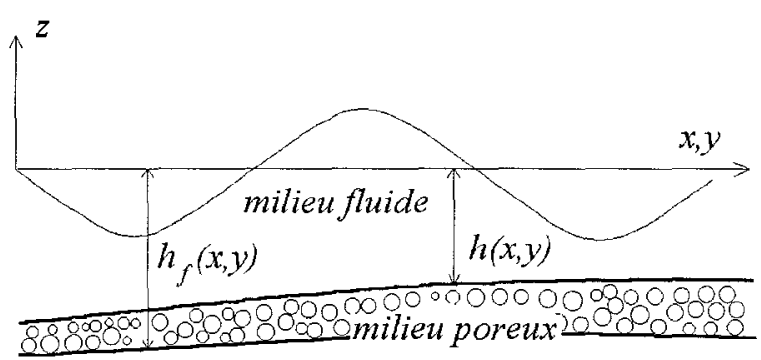

Figure 1 : Schéma du bicouche - schema of bilayer.

\subsection{Système d'équations}

Le modèle à pression qui régit la propagation d'une houle périodique en présence d'un fond poreux correspond au système d'équations suivant : 


$$
\left\{\begin{array}{lr}
\Delta \mathbf{P}=0 & -h \leq z \leq 0 \\
\operatorname{div}(\beta \vec{\nabla} \mathbf{P})=0 & -h_{f} \leq z \leq h \\
\frac{\partial \mathbf{P}}{\partial z}-\frac{\omega^{2}}{g} \mathbf{P}=0 & z=0 \\
\mathbf{P}\left(-h^{-}\right)=\mathbf{P}\left(-h^{+}\right) & z=-h \\
\vec{\nabla} \mathbf{P}\left(-h^{-}\right) \cdot \vec{n}=\beta \vec{\nabla} \mathbf{P}\left(-h^{+}\right) \cdot \vec{n} & z=-h \\
\beta \vec{\nabla} \mathbf{P} \cdot \vec{n}_{f}=0 & z=-h_{f}
\end{array}\right.
$$

\subsection{Relation de dispersion}

Considérons le cas de fonds plats avec $\beta$ constant dans la couche poreuse et écrivons la solution du système précédent sous la forme de variables séparées :

$$
\mathbf{P}=\phi(x, y) f(z)
$$

Dans la couche poreuse et la couche fluide, les deux premières équations du système précédent donnent : $-\frac{\Delta \phi}{\phi}=\frac{f^{\prime \prime}}{f}=k^{2}$

Le nombre d'onde $k$ est identique dans les deux couches. La solution sur la verticale se met sous la forme suivante avec quatre constantes $\mathrm{A}, \mathrm{B}, \mathrm{C}, \mathrm{D}$ :

$$
\begin{array}{ll}
f(z)=A \operatorname{ch}(k z)+B \operatorname{sh}(k z) & -h \leq z \leq 0 \\
f(z)=\mathrm{C} \operatorname{ch}(k z)+D \operatorname{sh}(k z) & -h_{f} \leq z \leq h
\end{array}
$$

L'application des conditions aux limites, sur la surface libre, le fond imperméable et à l'interface des deux milieux permet de calculer ces constantes ainsi que la relation de dispersion. Calculer la relation de dispersion consiste à déterminer une solution non nulle du système et à annuler le déterminant suivant :

$$
\text { Dét }\left|\begin{array}{cccc}
-\frac{\omega^{2}}{g k} & 1 & 0 & 0 \\
0 & 0 & -\operatorname{th}\left(k h_{f}\right) & 1 \\
1 & -\operatorname{th}(k h) & -1 & t h(k h) \\
-t h(k h) & 1 & \beta \operatorname{th}(k h) & -\beta
\end{array}\right|=0
$$

La relation de dispersion s'écrit: 


$$
\omega^{2}=g k t h(k h)+\beta \operatorname{th}\left(k\left(h_{f}-h\right)\right)\left(g k-\omega^{2} t h(k h)\right)
$$

On peut voir que pour $\left(h_{f}-h\right)$ nul, on retrouve l'équation pour une couche de fluide seule. Cette relation de dispersion a une infinité de valeurs propres solutions $k_{n}$ et de fonctions propres associées $f_{n}$. On démontre que ces fonctions propres sont orthogonales.

$$
\begin{gathered}
f_{n}(z)=\operatorname{ch}\left(k_{n} z\right)+\frac{\omega^{2}}{g k_{n}} \operatorname{sh}\left(k_{n} z\right) \quad-h \leq z \leq 0 \\
f_{n}(z)=\alpha \frac{\operatorname{ch}\left(k_{n}\left(z+h_{f}\right)\right)}{\operatorname{ch}\left(k_{n} h_{f}\right)}-h_{f} \leq z \leq-h \\
\text { avec } \alpha=\left(1-\frac{\omega^{2}}{g k_{n}} \operatorname{th}\left(k_{n} h\right)\right) \frac{\operatorname{ch}\left(k_{n} h\right) \operatorname{ch}\left(k_{n} h_{f}\right)}{\operatorname{ch}\left(k_{n}\left(h_{f}-h\right)\right)}
\end{gathered}
$$

La norme $F_{n}$ de ces fonctions est égale à :

$$
\begin{aligned}
F_{n} & =\beta \int_{-h_{f}}^{h} f_{n}^{2}(z) d z+\int_{-h}^{0} f_{n}^{2}(z) d z \\
& =\beta \alpha^{2} \frac{\operatorname{sh}\left(2 k_{n}\left(h_{f}-h\right)\right)+2 k_{n}\left(h_{f}-h\right)}{4 k_{n} c h^{2}\left(k_{n} h_{f}\right)}+\frac{1}{2}\left[h+\frac{\operatorname{sh}\left(2 k_{n} h\right)}{2 k_{n}}-\frac{\omega^{2} s h^{2}\left(k_{n} h\right)}{2 g k_{n}^{2}}+\frac{\omega^{4}\left\{s h\left(2 k_{n} h\right)-2 k_{n} h\right\}}{2 g^{2} k_{n}^{3}}\right]
\end{aligned}
$$

\subsection{Modèle horizontal}

Nous présentons un modèle incluant les ouvrages réfléchissants et moyenné sur la verticale. L'équation déduite représente un modèle horizontal dissipatif permettant de modéliser les problèmes de propagation d'une houle en présence de corps poreux. Ce modèle permet notamment de traiter la réflexion partielle de la houle sur les ouvrages. Cette démarche a déjà été entreprise par certains auteurs comme Sato et al. (1990) et Dalrymple et al. (1991). Les modèles utilisés par ces auteurs sont basés sur l'équation de pente douce avec un terme dissipatif supplémentaire. Sato propose d'ajouter une zone fictive de dissipation d'énergie devant les ouvrages réfléchissants, cette zone est caractérisée par un coefficient de dissipation. Dans une démarche similaire à la notre et sur le cas d'un obstacle poreux vertical à surface libre reposant sur un fond rigide variable, Zhu (2000) a présenté un modèle moyenné sur la hauteur de l'obstacle.

Dans ce qui suit, nous présentons la moyenne de la relation de propagation en milieu inhomogène sur une hauteur comprenant une couche poreuse et une couche fluide. 
Soit $f\left(z, h(x, y), h_{f}(x, y)\right)$ une fonction de pondération. Nous écrivons la forme faible suivante : $\left\{\begin{aligned} \int_{-h_{f}}^{h} f & \operatorname{div}(\beta \nabla \mathbf{P}) d z=0 \\ & \int_{-h}^{0} f \Delta \mathbf{P} d z=0\end{aligned}\right.$

Après intégration par parties et applications des conditions aux limites, on obtient :

$$
d i v\left(\beta \int_{-h f}^{-h} f \vec{\nabla} \mathbf{P} d z+\int_{-h}^{0} f \vec{\nabla} \mathbf{P} d z\right)-\beta \int_{-h f}^{-h} \vec{\nabla} f \cdot \vec{\nabla} \mathbf{P} d z-\int_{-h}^{0} \vec{\nabla} f . \vec{\nabla} \mathbf{P} d z-\beta \int_{-h f}^{-h} \frac{\partial f}{\partial z} \frac{\partial \mathbf{P}}{\partial z} d z-\int_{-h}^{0} \frac{\partial f}{\partial z} \frac{\partial \mathbf{P}}{\partial z} d z+\left[f \frac{\partial \mathbf{P}}{\partial z}\right]_{z=0}=0
$$

Soit la forme générale de la solution exprimée à partir d'une série de modes propres verticaux :

$$
\mathbf{P}(x, y, z)=\sum_{n=0}^{\infty} f_{n}(z, h, h f) \phi_{n}(x, y)
$$

Injectons cette forme et choisissons $f_{p}$ comme fonction de pondération. L'orthogonalité des fonctions $f_{n}$ donne la relation suivante :

$$
\begin{gathered}
\operatorname{div}\left(F_{P} \vec{\nabla} P_{p}\right)+\frac{\omega^{2}}{g} \sum_{n=0}^{\infty} P_{n}+\sum_{n=0}^{\infty}\left[\vec{Q}_{p n}-\vec{Q}_{n p}\right] \vec{\nabla} P_{n}+\sum_{n=0}^{\infty}\left[\operatorname{div} \vec{Q}_{p n}-R_{p n}-P_{p n}\right]_{n}=0 \\
\operatorname{avec}\left\{\begin{array}{cc}
F_{p n}=\beta \int_{-h f}^{-h} f_{p} f_{n} d z+\int_{-h}^{0} f_{p} f_{n} d z & R p n=\beta \int_{-h_{f}}^{-h} \vec{\nabla} f_{p} \cdot \vec{\nabla} f_{n} d z+\int_{-h}^{0} \vec{\nabla} f_{p} \cdot \vec{\nabla} f_{n} d z \\
P_{p n}=\beta \int_{-h_{f}}^{-h} \frac{\partial f_{p} \partial f_{n}}{\partial z} d z+\int_{-h}^{0} \frac{\partial f_{p} \partial f_{n}}{\partial z} d z & \vec{Q}_{p n}=\beta \int_{-h_{f}}^{-h} f_{p} \cdot \vec{\nabla} f_{n} d z+\int_{-h}^{0} f_{p} . \vec{\nabla} f_{n} d z
\end{array}\right.
\end{gathered}
$$

En négligeant la contribution de tous les modes évanescents d'ordre supérieur, nous récrivons la relation pour le premier mode $p=n=0$ :

$$
\operatorname{div}\left(F_{0} \vec{\nabla} P_{0}\right)+k_{0}^{2} F_{0} P_{0}=\left(R_{00}-\operatorname{div} \vec{Q}_{00}\right) P_{0}
$$

L'hypothèse des fonds et interfaces de faibles pentes permet de négliger le terme $R_{00}$ en $(\vec{\nabla} h)^{2}$ et $\left(\vec{\nabla} h_{f}\right)^{2}$ ainsi que $\operatorname{div} \vec{Q}_{00}$ en $\Delta h$ et $\Delta h_{f}$.

L'équation de pentes douces généralisée à des domaines avec fonds poreux dissipatifs s'écrit donc :

$$
\operatorname{div}\left(F_{0} \vec{\nabla} P_{0}\right)+k_{0}^{2} F_{0} P_{0}=0
$$


On vérifie que, pour des hauteurs de couches poreuses nulles, on retrouve l'équation classique de Berkhoff pour une couche fluide seule :

$$
\operatorname{div}\left(c c_{g} \vec{\nabla} P_{0}\right)+k_{0}^{2} c c_{g} P_{0}=0
$$

L'équation des pentes douces est résolue par éléments finis avec schéma itératif pour le calcul de $F_{0}$.

\section{Applications}

\subsection{Bosse poreuse triangulaire}

Les auteurs Isobe, Cruz et Watanabe (1997) ont développé un modèle horizontal temporel qui tient compte des non linéarités de la surface libre et du terme d'inertie pour étudier les fonds poreux. Le modèle repose sur les équations de Boussinesq appliquées aux fonds poreux. Ils ont appliqué notamment leur modèle sur les cas d'un fond poreux sur une bosse poreuse triangulaire. Les résultats obtenus par les auteurs ont été confrontés à des mesures expérimentales effectuées dans des bassins à houle à l'université de Tokyo. Nous avons testé notre modèle sur ce cas dont le profil et les dimensions géométriques du fond sont donnés sur la figure 2 .
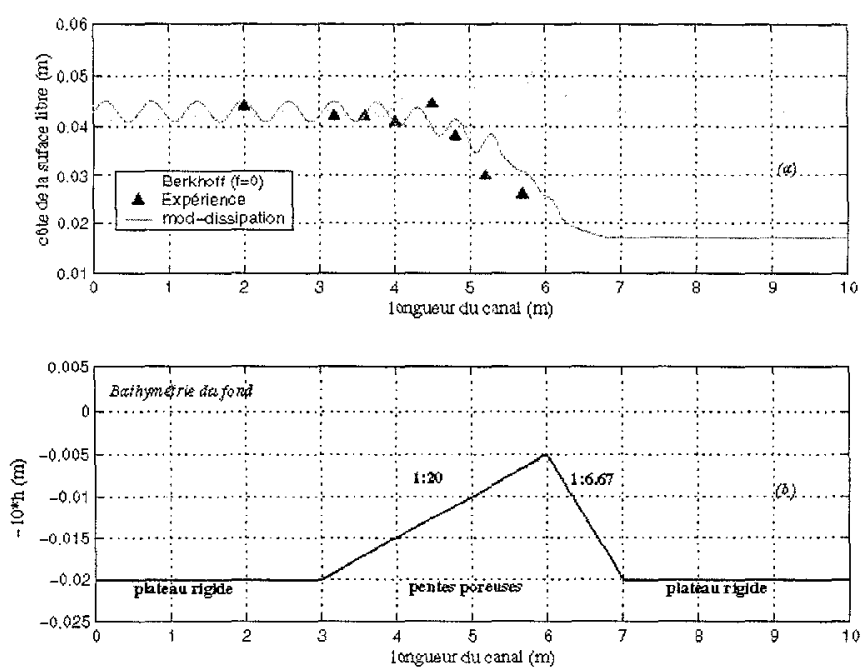

Figure 2 : Bosse poreuse triangulaire - triangular porous bar.

La houle est générée à l'extrémité $x=0$ et est absorbée de l'autre côté. Les résultats numériques obtenus sont représentés sur la figure 2 pour permettre une comparaison avec les mesures expérimentales. Afin de mieux voir l'amortissement de la houle lorsqu'elle traverse la bosse poreuse, nous avons représenté deux courbes, l'une représentant l'élévation de la surface libre dans le cas d'un fond poreux, l'autre est relative au cas d'un fond rigide. Nous pouvons comparer sur ces 
deux graphiques l'effet de l'ouvrage poreux sur la houle par rapport à celui d'un ouvrage rigide. On peut voir l'atténuation de la houle lorsque cette dernière se propage au dessus du corps poreux. On constate que la partie transmise de la houle est faible par rapport au cas où la pente était rigide. On remarque aussi que les résultats fournis par notre modèle sont proches des valeurs expérimentales. Les calculs correspondent à un diamètre équivalent $d$ égal à $0,67 \mathrm{~cm}$, une porosité $\varepsilon$ égale à 0,44 , un coefficient de frottement $C_{f}$ égal à 0,40 et une perméabilité $K$ égale à $2,510^{-4} \mathrm{~cm}^{2}$.

\subsection{Digue à talus}

Les caractéristiques physiques de l'enrochement d'une digue à talus sont prises des valeurs expérimentales présentées par Sollit et Cross (1972) et reprises par Madsen (1974). Elles correspondent à un diamètre équivalent $d$ égal à $1 \mathrm{~cm}$, la porosité $\varepsilon$ égal à 0,43 , le coefficient de frottement $C_{f}$ égal à 0,41 et la perméabilité $K$ égale à $3,710^{-4} \mathrm{~cm}^{2}$.

Le tableau 1 représente les valeurs numériques du coefficient de réflexion obtenu avec notre modèle, les valeurs fournies par la relation de Seelig ajustée prenant en compte notamment le diamètre des enrochements et par la relation par défaut de Seelig avec $(a ; b)=(0,6 ; 6,6)$. On constate que les résultats du modèle sont proches de la relation de Seelig ajustée. Pour les grands nombres d'Irribaren, la relation de Seelig est dite conservative car elle a tendance à sous-estimer le coefficient de réflexion. Les valeurs numériques obtenues sont légèrement supérieures à celles de Seelig.

\begin{tabular}{|c|c|c|c|c|}
\hline Période $(\mathrm{s})$ & $\begin{array}{c}\text { Nombre } \\
\text { d'Irribaren }\end{array}$ & $\begin{array}{c}\mathrm{K}_{\mathrm{R}} \\
\text { numérique }\end{array}$ & $\begin{array}{c}\mathrm{K}_{\mathrm{R}} \\
\text { Seelig ajusté }\end{array}$ & $\begin{array}{c}\mathrm{K}_{\mathrm{R}} \\
\text { Seelig }(0,6 / 6,6)\end{array}$ \\
\hline 0,5 & 2,94 & 0,44 & 0,40 & 0,34 \\
\hline 1 & 4,86 & 0,49 & 0,62 & 0,47 \\
\hline 2 & 6,13 & 0,73 & 0,76 & 0,51 \\
\hline 3 & 7,36 & 0,91 & 0,80 & 0,53 \\
\hline 4 & 8,44 & 0,93 & 0,82 & 0,55 \\
\hline 5 & 9,41 & 0,93 & 0,88 & 0,56 \\
\hline 6 & 10,29 & 0,93 & 0,85 & 0,56 \\
\hline
\end{tabular}

Tableau 1 : Comparaison des coefficients de réflexion pour une digue à talus comparison of reflection coefficients of a rubble mound breakwater.

\section{Conclusions}

Un modèle de houle à dissipation a été développé dans le but d'améliorer la prise en compte des ouvrages réfléchissants dans les codes de calcul d'agitation portuaire. Les résultats obtenus montrent que les ouvrages poreux réfléchissants peuvent bien être pris en compte comme une couche dissipative dont les 
caractéristiques sont déduites de la bathymétrie et de la géométrie des enrochements. Les calculs ont été développés ici pour un bicouche mais peuvent être facilement généralisés pour un multicouche.

\section{Références}

Cruz E.C., Isobe M. et Watanabe A., - Boussinesq equations for wave transformation on porous beds - Coast. Eng., 30, 125-156, 1997

Dalrymple R.A., Losada M.A. et Martin P.A. - Reflection and transmission from porous structures under oblique wave attack - Journal of fluid mechanics 224, 625-644, 1991

Dingemans M.W. -Water wave propagation over uneven bottoms- "World Scientific", 1996

Madsen O.S.- Wave transmission through porous structures - Coast. Eng. - 100 WW3, 169-188, 1974

Ropert F. - Modélisation numérique du comportement hydraulique des caissons Jarlan - PhD Thésis -Université Technologique de Compiègne, 1999

Sato W., Isobe M. et Isumiya T- A numerical Model for calculating wave height distribution in a harbour of arbitrary shape - Coast. Eng.- 33, 2, 119-131, 1990

Sollit C.K. et Cross R.H. - Wave transmission through permeable breakwater Coast. Eng. - 13, 1827-1846, 1972

Sulisz W. - Wave loads on caisson founded on multilayered rubble base, J. Wtrwy. Port Coast. and Oc. Eng. - 123, 3, 91-101,1997

Sulisz W. - Wave reflection and transmission at permeable breakwaters of arbitrary cross section - Coast. Eng.- 9, 4, 371-386, 1985

Ward J. C. -Turbulent flow in porous media - Proc. Asce. J. Hyd.- 90, 5, 1-12, 1964

Williams A.F., Burchart H.F. et Den Adel H. -The permeability of rubble mound breakwater and new ideas - Mast G6-S Coastal Structures - 2, 129138,1992

Zhu S.- On calculating the length of a short-crested over a porous seabed, Applied Ocean Research, 22, 63-73, 2000

Zhu S.-Water waves within a porous medium on an ondulating bed - Coast. Eng. $-42,87-101,2001$ 